\title{
BLADDER CONTRACTURE FOLLOWING INTRAVESICAL BCG IMMUNOTHERAPY
}

\author{
H. BARTON GROSSMAN, M.D.
}

From the Department of Surgery, Section of Urology, University of Michigan Medical Center, Ann Arbor, Michigan

\begin{abstract}
Intravesical BCG immunotherapy is commonly associated with irritative bladder symptoms. Bladder contracture as a result of this treatment is rare. Two cases of persistent small-capacity bladders occurring more than one year after intravesical BCG therapy are reported.
\end{abstract}

Bacillus Calmette-Guerin (BCG) has documented efficacy in the treatment of superficial bladder cancer. ${ }^{1}$ However, local toxicity with this agent tends to be greater than that found with other commonly employed intravesical chemotherapeutic agents, i.e., thiotepa, doxorubicin, and mitomycin-C. ${ }^{2}$ In a review of 1,278 patients, Lamm and associates ${ }^{3}$ reported that intravesical irritative symptoms (frequency and dysuria) were common and occurred in over 90 percent of the patients. Significant toxicity was uncommon, and in only 2 patients a contracted bladder developed. Both of these patients received the Tice strain on a monthly maintenance schedule without isoniazid prophylaxis. Of the five strains of BCG reviewed, the Connaught strain had the lowest incidence of complications. This report describes 2 patients receiving intravesical BCG (Connaught strain) in whom long-term bladder contraction developed as a result of their therapy.

\section{Material and Methods}

From September 30, 1983 through March 30 , 1988,49 patients with superficial bladder cancer were treated with intravesical BCG. Most of these patients were enrolled in one of several Southwest Oncology Group trials and werc treated with Connaught strain BCG. The treatment course usually consisted of intravesical and percutaneous administration of $120 \mathrm{mg}$ BCG weekly for six weeks. Some patients also received subsequent additional maintenance treatments with BCG.
Results

In our group of patients irritative symptoms of frequency and dysuria following intravesical BCG were common. No patient suffered systemic toxicity. One patient had significant voiding abnormalities with a small-capacity bladder that resolved over a period of five months. Two patients have had contracted bladders that have persisted for over one year. Their clinical courses are described.

\section{Case 1}

A seventy-four-year-old woman began her BCG therapy in July 1986 . She had a history of recurrent superficial bladder cancer dating back to 1982. Prior to starting BCG therapy, she had multiple transurethral resections of grade 1-2 papillary transitional cell carcinomas. In addition, she had previously failed treatment with intravesical thiotepa, Adriamycin, and mitomycin-C. Her last intravesical chemotherapy treatment (with mitomycin-C) was in January 1986.

Cystoscopy on July 7, 1986 demonstrated another grade 1-2, papillary transitional cell carcinoma. On July 14, 1986 she started a sixweek course of Connaught BCG (intravesical and percutaneous). Despite gross hematuria, frequency, and urgency, she elected to complete her treatment course. One month later her symptoms persisted, and she was given isoniazid $300 \mathrm{mg}$ daily for thirty days. An abdominal film demonstrated bladder calcification (Fig. 1). In October, cystoscopy 


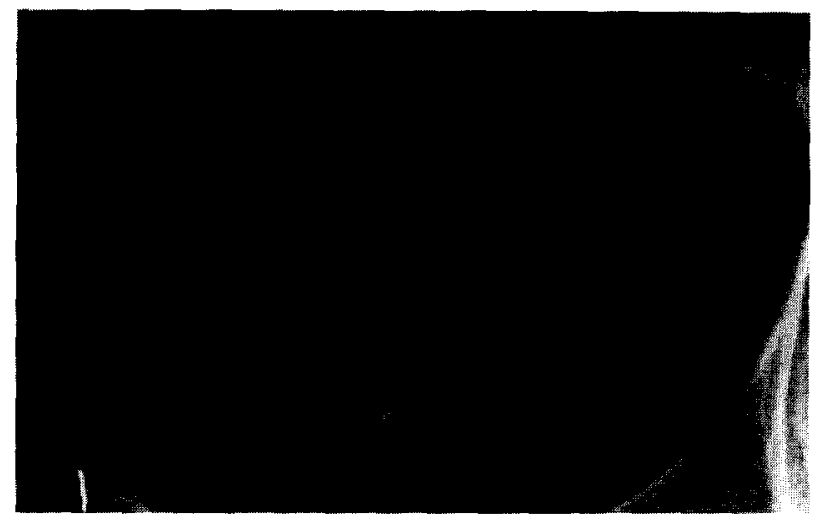

FIGURE 1. Radiograph demonstrating calcification.

demonstrated a grossly inflamed bladder with multiple areas of calcification of the bladder wall. Biopsy specimen demonstrated chronic inflammation. In June, several papillary grade 2 transitional cell carcinomas were resected along with another tumor just inside the left ureteral orifice. Her bladder was small and chronically inflamed. In July and October 1987, she had neodymium:yttrium-aluminumgarnet (Nd:YAG) laser therapy of the tumor inside the left ureteral orifice. Since that time, ureteroscopic examination showed her ureter has been tumor free. She had another bladder tumor that had laser therapy in January 1988. At her last follow-up in April 1988, she had a 70-mL capacity bladder with calculous cystitis on the posterior bladder wall (Fig. 2). The calculous material was easily removed with a biopsy forceps. Ureteroscopic examination demonstrated no tumor recurrence. Bladder biopsy specimen demonstrated chronic inflammation.

\section{Case 2}

A sixty-five-year-old woman started intravesical BCG in September 1986. She had a history of prior pelvic radiation and surgery in 1976 for carcinoma of the endometrium. Her first bladder tumor was in 1984. She had several recurrences (grade 2, noninvasive) and was started on a course of intravesical and percutaneous BCG on September 16, 1986. She received six weekly treatments with little toxicity. Followup cystoscopy and biopsy in December 1986 demonstrated chronic granulomatous cystitis. On December 16, she began a maintenance schedule of three weekly treatments with BCG. After her last treatment, she had a flu-like syndrome, mild urgency, and nocturia.

Follow-up cystoscopy in March demonstrated bladder erythema with distention. On

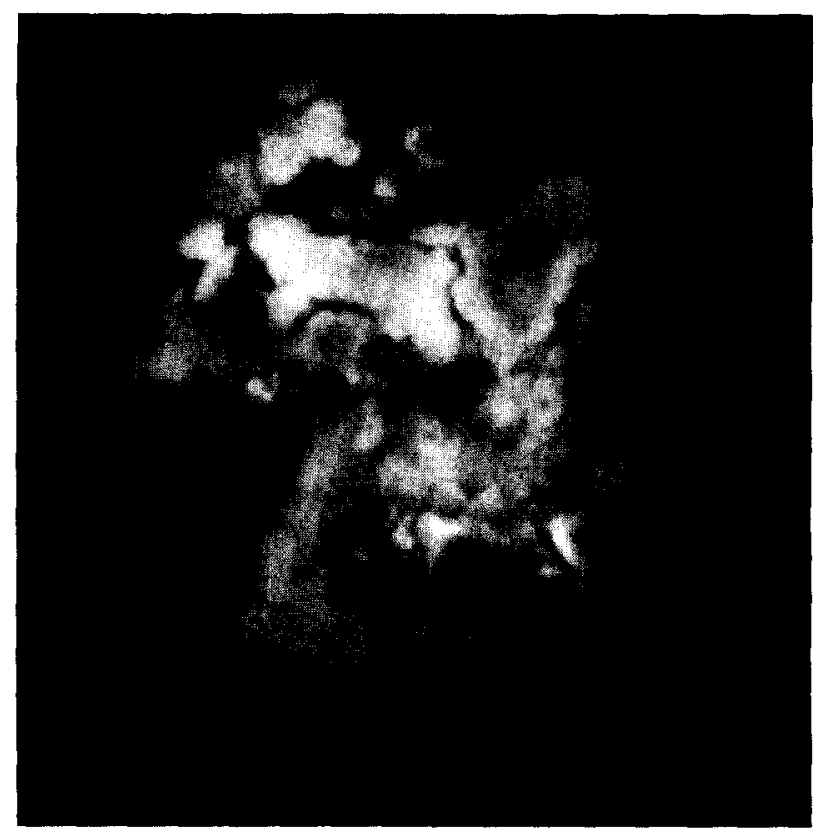

FIGURE 2. Endoscopic photograph demonstrating superficial bladder calcification.

March 24, 1987, she began another maintenance cycle of BCG. After her first dose, a mild flu-like syndrome developed along with urgency and frequency. After her second dose, a flu-like syndrome again developed. In addition, urgency and frequency were much worse and caused her to void hourly. She was not given her scheduled third treatment with BCG. In June 1987, cystoscopy demonstrated diffuse inflammation with patchy areas of hemorrhage. Her bladder capacity was $50 \mathrm{~mL}$. In September 1987, cystoscopy demonstrated a bladder capacity of $100 \mathrm{~mL}$. Distention to this level caused diffuse bleeding. A biopsy specimen showed marked chronic inflammation. An excretory urogram (IVP) demonstrated a smallcapacity bladder. Follow-up cystoscopic examinations in December 1987, March 1988, and June 1988 demonstrated no neoplasms. Bladder washings for cytologic examination were negative. Her bladder capacity had increased to $200 \mathrm{~mL}$, but at that volume she had pain and diffuse hemorrhage.

\section{Comment}

Intravesical BCG has been remarkably effective in preventing superficial bladder tumor recurrence $^{l}$ and progression to high-stage disease. ${ }^{4}$ However, accompanying this therapeutic efficacy is a high incidence of minor to moderate local toxicity. ${ }^{3}$ Less commonly, more significant toxicities have occurred. These include 
bladder outlet obstruction from granulomatous prostatitis, ${ }^{5}$ epididymitis, ${ }^{6}$ granulomatous lymphadenitis with ureteral obstruction, ${ }^{5}$ granulomatous hepatitis, ${ }^{6}$ and tuberculous pneumonia. ${ }^{7}$ The incidence of each of these major complications is 0.3 percent or less. ${ }^{3}$

The 2 patients with contracted bladders in this report attest to the fact that other strains of BCG besides Tice may cause this complication. Both of these patients had received previous therapy which may have contributed to their problems. Case 1 had failed therapy with multiple other intravesical chemotherapeutic agents and a calculous cystitis developed that ultimately resulted in bladder contracture. BCG does cause increased bacterial adherence and is associated with an increased incidence of urinary tract infections. ${ }^{8}$ This effect coupled with a heavily pretreated bladder may have resulted in this severe response.

Case 2 had been treated with pelvic radiation ten years earlier. She tolerated an initial course of therapy with minimal morbidity. However, her second course of maintenance therapy caused severe symptoms resulting in stopping treatment after 2 instillations. The progressive increase in symptoms with subsequent treatments strongly suggests an immune basis for this phenomenon. A hypersensitivity phenomenon from intravesical BCG also has caused interstitial pneumonitis. ${ }^{9}$

BCG remains an effective treatment modality for superficial bladder cancer. However, it is important to be aware of the possibility of severe toxicity from this treatment. Individuals who have significant local symptoms should be monitored carefully. There is a small but significant risk of long-term local morbidity and mortality from intravesical BCG immunotherapy. ${ }^{10}$

2916G Taubman Center Box 0330

Ann Arbor, Michigan 48109

\section{References}

1. Lamm DL, Thor DE, Stogdill VD, and Radwin HM: Bladder cancer immunotherapy, J Urol 128: 931 (1982).

2. Soloway MS: Introduction and overview of intravesical therapy for superficial bladder cancer, Urology (Suppl 3) 31: 5 (1988).

3. Lamm DL, Stodgill VD, Stodgill BJ, and Crispen RG: Complications of bacillus Calmette-Guerin immunotherapy in 1,278 patients with bladder cancer, J Urol 135: 272 (1986).

4. Herr HW, et al: Long-term effect of intravesical bacillus Calmette-Guerin on flat carcinoma in situ of the bladder, J Urol 135: 265 (1986).

5. Orihuela E, Herr HW, Pinsky CM, and Whitmore WF Jr: Toxicity of intravesical BCG and its management in patients with superficial bladder tumors, Cancer 60: 326 (1987).

6. Steg A, Leleu C, Boccon-Gibod L, and Debre B: Traitement des tumeurs superficielles et des carcinomes in situ de vessie par BCG-therapie intravesicale, Ann Urol 20: 26 (1986).

7. Haaf EO, Dresner SM, Ratliff TL, and Catalona WJ: Two courses of intravesical bacillus Calmette-Guerin for transitional cell carcinoma of the bladder, J Urol 136: 820 (1986).

8. Bruce AW, et al: Adherence of gram-negative uropathogens to human uroepithelial cells, J Urol 130: 293 (1983).

9. Israel-Biet $\mathrm{D}$, et al: Pulmonary complications of intravesical bacillus Calmette-Guerin immunotherapy, Am Rev Respir Dis 135: 763 (1987).

10. Rawls WH, Lamm DL, and Eyolfson MF: Septic complications in the use of bacillus Calmette-Guerin (BCG) for non invasive transitional cell carcinoma (Abstr. 552), J Urol 139: 300A (1988). 